\title{
Efectividad antiinflamatoria del eugenol frente al croton lechleri en el tratamiento de la estomatitis aftosa recurrente
}

\author{
Anti-inflammatory effectiveness of eugenol compared to croton lechleri in the \\ treatment of recurrent aphthous stomatitis
}

Marisol R. Ortega Buitrón ${ }^{1, a *}$, Nancy D. Calzada Gonzales ${ }^{1, a, c}$

Filiación y grado académico

Universidad de Huánuco, Perú (Docente auxiliar).

a Cirujano Dentista/Doctor, Huánuco, Perú.

b Cirujano Dentista.

' Doctora en Ciencias de la Salud.

ORCID iD de Marisol R. Ortega Buitrón https://orcid.org/0000-0001-6283-2599

(10) ORCID iD de Nancy D. Calzada Gonzales https://orcid.org/0000-0001-5179-3790

Contribución de los autores

MOB: ejecución de la investigación y elaboración del artículo.

NCG: ejecución de la investigación y análisis estadístico.

Fuentes de financiamiento Universidad de Huánuco.

Conflictos de interés

Las autoras declaran no tener conflictos de interés en la publicación de este artículo.

Recibido: 02/11/2018

Arbitrado por pares

Aceptado: 04/02/2019

Citar como

Ortega MR, Calzada N. Efectividad antiinflamatoria del eugenol frente al croton lechleri en el tratamiento de la estomatitis aftosa recurrente. Rev Peru Cienc Salud. 2019 (1): 30-5. doi: https://doi. org/10.37711/rpcs. 2019.1.1.3

Correspondencia

Marisol Rossana, Ortega Buitrón

Cel.: 942586 492/062503052

Email: marisolrosana@hotmail.com

\section{RESUMEN}

Objetivo. Determinar la efectividad antiinflamatoria del eugenol frente al croton lechleri en el tratamiento de la estomatitis aftosa recurrente. Métodos. Se trata de un estudio experimental donde fueron incluidos 60 muestras, divididas en tres grupos de estudio (G.E): G.E. 1 (croton lechleri), con 20 sujetos; G.E. 2 (eugenol), con 20 sujetos; y el grupo control (G.C), con otros 20 sujetos. Se evaluaron las características clínicas, el dolor, el eritema, el tiempo de cicatrización; así como el recuento de los leucocitos y la proteína Creactiva, antes y dos días después de la aplicación del tratamiento. Se utilizó la prueba t de Student, la prueba de Kruskal-Wallis y la U de Mann-Whitney. Resultados. Las aftas menores presentaron mayor frecuencia, con el 93,3\%; la forma redonda predominó en los sujetos estudiados, con un $63,3 \%$; con un $28,3 \%$ en varias localizaciones; seguidas de la lengua, con el $25 \%$, y la mucosa labial, con el 16,7 \%. El dolor cesó al $100 \%$ en los pacientes al segundo día de aplicado el eugenol, el eritema al tercer día y la cicatrización culminó al cuarto día. Con el uso del croton lechleri, al tercer día cesó el dolor y el eritema, culminando el proceso de cicatrización. Conclusión. Los resultados del presente estudio demuestran que tanto la aplicación tópica de croton lechleri, como la del eugenol, son efectivos en el tratamiento de la estomatitis aftosa recurrente.

Palabras clave: enfermedad de Sutton 2, Estomatitis aftosa, Eugenol, Proteína C-reactiva, Croton, Eritema, Estadísticas, no paramétrico, Grupos de control, Enfermedades de la lengua, Lengua, Boca, mucosa, Labio, Leucocitos, Agentes antiinflamatorios, Dolor (Fuente: DeCS-Bireme).

\section{ABSTRACT}

Objective. To determine the anti-inflammatory effectiveness of eugenol against croton lechleri in the treatment of recurrent aphthous stomatitis. Methods. This is an experimental study where 60 samples were included, divided into three study groups (G.E): G.E. 1 (croton lechleri), with 20 subjects; G.E. 2 (eugenol), with 20 subjects; and the control group (G.C), with 20 other subjects. The clinical characteristics, pain, erythema, healing time were evaluated; as well as the count of leukocytes and C-reactive protein, before and two days after the application of the treatment. The Student t-test, the Kruskal-Wallis test and the Mann-Whitney U test were used. Results. Minor canker sores were more frequent, with $93.3 \%$; the round shape predominated in the subjects studied, with $63.3 \%$; with $28.3 \%$ in several locations; followed by the tongue, with $25 \%$, and the labial mucosa, with $16.7 \%$. Pain ceased $100 \%$ in patients on the second day of applying eugenol, erythema on the third day and healing ended on the fourth day. With the use of croton lechleri, on the third day the pain and erythema ceased, culminating the healing process. Conclusions. The results of the present study demonstrate that both the topical application of croton lechleri, and that of eugenol, are effective in the treatment of recurrent aphthous stomatitis.

Keywords: sutton disease 2, Stomatitis Aphthous, Eugenol, C-Reactive Protein, Croton, Erythema, Statistics, Nonparametric, Control Groups, Tongue Diseases, Tongue, Mouth Mucosa, Lip, Leukocytes, Anti-Inflammatory Agents, Pain (Source: MeSH-MedLine). 


\section{INTRODUCCIÓN}

Las aftas o estomatitis aftosa recurrente (EAR) son úlceras vesiculares muy dolorosas, de forma oval, de un diámetro menor a $0,5 \mathrm{~cm}$ envueltas por un tipo de membrana fibrinosa amarillenta y un halo eritematoso a su alrededor; presentándose de forma única aunque en determinadas ocasiones de forma múltiple. La etiología de estas lesiones sigue siendo de origen desconocido. Se le atribuye una causa multifactorial así como una posible base genética, habiendo también la existencia de otros factores predisponentes como el trauma, el estrés, el consumo de determinados alimentos, el desequilibrio hormonal y el tabaco; encontrándose asimismo otros factores predisponentes como la intervención de virus y bacterias, las deficiencias vitamínicas y los factores inmunológicos. Van a existir tres formas clínicas de aparición como las lesiones aftosas menores, las aftosas mayores y la estomatitis aftosa herpetiforme. Finalmente, emergen una serie de síndromes que cursan con aparición de aftas a nivel oral, denominándose síndromes aftosos. Al no conocer la etiología y la naturaleza cíclica del proceso complican el tratamiento. Por este motivo se sugirió numerosas y diversas terapias contando con una amplia literatura al respecto pero con resultados inconsistentes. El tratamiento debe ser diseñado de manera individual y sintomática, persiguiendo objetivos principales como: disminuir el proceso, evitar recidivas y reducir los síntomas como el tamaño de las úlceras durante el brote ${ }^{(1)}$.

El eugenol es un derivado fenólico conocido comúnmente como "esencia de clavo" que es utilizado desde hace varios siglos en la práctica odontológica. Por sus propiedades farmacológicas tiene diferentes usos. Sus efectos farmacológicos son complejos y dependen de la concentración del eugenol libre al que se expone el tejido ${ }^{(2)}$.

Torres Lagares encontró que la efectividad del eugenol enforma de pastas antisépticas intraalveolares medicamentosas en casos de alveolitis, acaban dando buenos resultados. Estas pastas medicamentosas, según su principio activo, se pueden clasificar en apósitos antimicrobianos, apósitos calmantes o apósitos con anestésicos locales. En un estudio publicado por Garibaldi et ál., se comparó la ventaja del tratamiento con apósitos a base de eugenol, de lidocaína y de enjuague con clorhexidina al 0,12\%, encontrando que el primero de ellos produce una mayor reducción del tiempo de curación, por lo cual puede tener acción también en las aftas bucales ${ }^{(3)}$.
Así mismo, la presencia del croton lechleri, cuyo nombre popular es "sangre de grado" en la selva peruana y específicamente en parte de nuestra región, puede ser una alternativa ya que esta planta tiene acción analgésica, antiinflamatoria y cicatrizante para el estómago y la piel; entre otras partes del cuerpo ${ }^{(4)}$.

La sangre de grado se usa en el proceso de cicatrización de las úlceras gástricas y de todo tipo de heridas, por lo que se tendría motivos suficientes para aplicar este producto en el tratamiento de las aftas de la mucosa bucal ${ }^{(4)}$.

Desde hace muchos años, las úlceras aftosas han significado un problema cotidiano y muy común dentro de las patologías bucales. Tal vez sean las que han merecido una menor importancia, por su remisión, teniendo una duración de 7 a 10 días y la no existencia de un tratamiento eficaz y específico, afectando el libre desenvolvimiento y produciendo un dolor agudo, remitente y molesto. Si se disminuye los días en la curación disminuyen los riesgos para las complicaciones de estas ulceras orales, ya que estas constituyen una puerta de entrada para las infecciones oportunistas ${ }^{(5)}$.

Si se trabaja para disminuir el dolor, la inflamación y el número de días en la cicatrización de las heridas aftosas recurrentes de la mucosa bucal, sería un gran aporte a la ciencia por que se disminuiría los días expuesta al riesgo de infección y se aliviaría al paciente mejorando su calidad de vida ${ }^{(6)}$.

Según las propiedades curativas de la sangre de grado (croton lechleri) podría ser una alternativa para disminuir esta problemática ${ }^{(7)}$. La sangre de grado (croton lechleri) es una especie forestal amazónica que en los últimos años ha acrecentado su demanda en el mercado nacional e internacional, debido a sus propiedades medicinales atribuidas al látex en el proceso de cicatrización de heridas y en el tratamiento de afecciones estomacales como úlceras ${ }^{(7)}$.

El uso del croton lechleri, en la solución de los problemas de salud corresponde al bagaje de experiencias de las poblaciones indígenas de la Amazonía peruana. En América del Sur (Bolivia, Colombia y Ecuador) y en el Perú se encuentran en los departamentos de Loreto, San Martín, Huánuco, Junín, Puno y Pasco ${ }^{(8,9)}$.

La aplicación de sangre de grado en el campo de la estomatología con extractos puros, de forma tópica o formando parte de un cemento clínico, llevó al inicio de los procesos reparativos, regenerativos 
Tabla 1. Distribución de las lesiones según forma clínica en grupos de estudio

\begin{tabular}{|c|c|c|c|c|c|c|c|c|}
\hline & \multicolumn{2}{|c|}{ Eugenol } & \multicolumn{2}{|c|}{ Croton L. } & \multicolumn{2}{|c|}{$\begin{array}{c}\text { Agua } \\
\text { destilada }\end{array}$} & \multicolumn{2}{|c|}{ Total } \\
\hline & fi & $\%$ & fi & $\%$ & fi & $\%$ & $\mathrm{fi}$ & $\%$ \\
\hline Menor & 21 & 35,0 & 18 & 30,0 & 17 & 28,3 & 56 & 93,3 \\
\hline Mayor & 2 & 3,3 & 1 & 1,7 & 1 & 1,7 & 4 & 6,7 \\
\hline Herpetiforme & 0 & 0,0 & 0 & 0,0 & 0 & 0,0 & 0 & 0,0 \\
\hline Total & 23 & 38,3 & 19 & 31,7 & 18 & 30,0 & 60 & 100,0 \\
\hline
\end{tabular}

y cicatrizantes de los tejidos orales a los cuales se aplicó ${ }^{(10,11)}$. También tuvo resultados significativos en el proceso de cicatrización de las heridas pos exodoncia, en un tiempo promedio de 18 a 20 días; lo que normalmente demora en cicatrizar alrededor de 30 días ${ }^{(12)}$.

La distribución mundial de las aftas (EAR) es realmente preocupante, ya que por lo menos un $80 \%$ de la población del mundo las ha padecido. Dentro de este grupo, entre un 10 y un $55 \%$ de la población, aumenta de acuerdo al nivel educativo, socioeconómico e historia familiar siendo, las más vulnerables las mujeres en un $55 \%$ frente al $45 \%$ de los hombres que la padecen. En relación a la edad cualquiera puede padecerlas, pero es más frecuente en personas entre los 20 y los 30 años de edad ${ }^{(6)}$.

En este estudio se determinó la efectividad antiinflamatoria del eugenol frente al croton lechleri en el tratamiento de la EAR.

\section{MÉTODOS}

Se trata de un estudio experimental. La muestra estuvo constituida por 60 pacientes con diagnóstico de EAR que cumplieron con los criterios de elegibilidad y fueron atendidos en el consultorio de odontología del Hospital Regional Hermilio Valdizán.

Como criterios de inclusión se abarcaron pacientes mayores, de entre 20 y 60 años de edad; quienes
Tabla 2. Distribución de las estomatitis aftosas recurrentes según forma de la lesión en grupos de estudio

\begin{tabular}{|c|c|c|c|c|c|c|c|c|}
\hline & \multicolumn{2}{|c|}{ Eugenol } & \multicolumn{2}{|c|}{ Croton L. } & \multicolumn{2}{|c|}{$\begin{array}{c}\text { Agua } \\
\text { destilada }\end{array}$} & \multicolumn{2}{|c|}{ Total } \\
\hline & fi & $\%$ & fi & $\%$ & $f i$ & $\%$ & fi & $\%$ \\
\hline Redondeada & 14 & 23,3 & 13 & 21,7 & 11 & 18,3 & 38 & 63,3 \\
\hline Oval & 5 & 8,3 & 4 & 6,7 & 5 & 8,3 & 14 & 23,3 \\
\hline Irregular & 3 & 5,0 & 3 & 5,0 & 2 & 3,4 & 8 & 13,4 \\
\hline Total & 22 & 36,6 & 20 & 33,4 & 18 & 30,0 & 60 & 100,0 \\
\hline
\end{tabular}

firmaron el formato de consentimiento informado En la fase experimental primero se desinfectó la zona lesionada con clorhexidina al $0,12 \%$ en los tres grupos de estudio, para el G.E. 1 se aplicó el crotón lechleri dos gotas en la lesión de igual manera se aplicó 2 gotas de eugenol en la zona de la lesión cada 8 horas por 5 días y para el G.C., se aplicó dos gotas de agua destilada. Una vez aplicado el tratamiento se evaluaron las características clínicas, el dolor, el eritema y el tiempo de cicatrización. Además se hizo un recuento de leucocitos y proteína $C$ Reactiva en sangre, antes y después de los 2 días de haberse aplicado el tratamiento. Las pruebas estadísticas utilizadas a través del programa estadístico SPSS (versión 23) para el análisis de los resultados fueron descriptivas (medias y desviación estándar).

\section{RESULTADOS}

En la tabla 1 se observa la distribución de las lesiones según su forma clínica en los grupos de estudio evaluados. Las aftas menores se presentaron con mayor frecuencia en un 93,3\% predominando en los tres grupos de estudio, seguida de las aftas mayores $6,7 \%$, no encontrándose casos de formas herpetiformes.

En la tabla 2 se observa la distribución las lesiones en los grupos de estudio evaluados, de acuerdo a la forma que presenta la lesión; la forma redonda predominó en los sujetos estudiados con un $63,3 \%$, seguida de la forma oval, con un $23,3 \%$, seguida de la forma irregular, con un 13,4\%.

Tabla 3. Tiempo de desaparición de las características clínicas de la estomatitis aftosa recurrente con el uso del Eugenol

\begin{tabular}{|c|c|c|c|c|c|c|c|c|c|c|c|c|}
\hline \multirow{3}{*}{ Dias } & \multicolumn{4}{|c|}{ Dolor } & \multicolumn{4}{|c|}{ Eritema } & \multicolumn{4}{|c|}{ Tiempo de cicatrización } \\
\hline & \multicolumn{2}{|c|}{ Sí } & \multicolumn{2}{|c|}{ No } & \multicolumn{2}{|c|}{ Sí } & \multicolumn{2}{|c|}{ No } & \multicolumn{2}{|c|}{ Sí } & \multicolumn{2}{|c|}{ No } \\
\hline & fi & $\%$ & fi & $\%$ & fi & $\%$ & fi & $\%$ & $\mathrm{fi}$ & $\%$ & fi & $\%$ \\
\hline Día 1 & 20 & 100,0 & 0 & 0,0 & 20 & 100,0 & 0 & 0,0 & 20 & 100,0 & 0 & 0,0 \\
\hline Día 2 & 0 & 0,0 & 20 & 100,0 & 9 & 45,0 & 11 & 55,0 & 5,0 & 25,0 & 10 & 50,0 \\
\hline Día 3 & & & & & 0 & 0,0 & 20 & 100,0 & 3,0 & 15,0 & 17 & 85,0 \\
\hline Día 4 & & & & & & & & & 0,0 & 0,0 & 20 & 10,0 \\
\hline
\end{tabular}


Tabla 4. Tiempo de desaparición de las características clínicas de la estomatitis aftosa recurrente con el uso del Croton Lechleri

\begin{tabular}{|c|c|c|c|c|c|c|c|c|c|c|c|c|}
\hline \multirow{3}{*}{ Dias } & \multicolumn{4}{|c|}{ Dolor } & \multicolumn{4}{|c|}{ Eritema } & \multicolumn{4}{|c|}{ Tiempo de cicatrización } \\
\hline & \multicolumn{2}{|c|}{ Sí } & \multicolumn{2}{|c|}{ No } & \multicolumn{2}{|c|}{ Sí } & \multicolumn{2}{|c|}{ No } & \multicolumn{2}{|c|}{ Sí } & \multicolumn{2}{|c|}{ No } \\
\hline & fi & $\%$ & fi & $\%$ & fi & $\%$ & fi & $\%$ & fi & $\%$ & fi & $\%$ \\
\hline Día 1 & 20 & 100,0 & 0 & 0,0 & 20 & 100,0 & 0 & 0,0 & 20 & 100,0 & 0 & 0,0 \\
\hline Día 2 & 7 & 35,0 & 13 & 65,0 & 10 & 50,0 & 10 & 50,0 & 10 & 50,0 & 10 & 100,0 \\
\hline Día 3 & 0 & 0,0 & 20 & 100,0 & 0 & 0,0 & 20 & 100,0 & 0 & 0,0 & 20 & 100,0 \\
\hline
\end{tabular}

En la tabla 3 se evidencia el tiempo de desaparición del dolor en la estomatitis recurrente con el uso del eugenol. El $100 \%$ de los sujetos de estudio manifestaron que en el segundo día cesó el síntoma. En el tiempo de desaparición del eritema de la estomatitis recurrente con el uso del eugenol, en los sujetos de estudio se observó que al tercer día no presentaron de características eritematosas; mientras que, en el segundo día, el $45 \%$ de los pacientes continuaban con dicho signo clínico. En el tiempo de cicatrización de la estomatitis recurrente aftosa con el uso del eugenol, en los sujetos de estudio se observó que al tercer día, la lesión presentaba características clínicas de cicatrización; mientras que en el segundo día, el $15,0 \%$ de los pacientes presentaba características del proceso de cicatrización; finalmente, el cuarto día, el total de los sujetos estudiados culminaron el proceso de cicatrización.

En el tiempo de desaparición del dolor de la estomatitis recurrente aftosa con el uso del croton lechleri, los sujetos de estudio no presentaron dolor en el tercer día, mientras que en el segundo día el $35 \%$ de los pacientes manifestaron dolor. (ver tabla 4) En el tiempo de desaparición del eritema de la EAR con el uso del croton lechleri, en los sujetos de estudio se observó que al tercer día, no presentaron características eritematosas; mientras que en el segundo día el $50 \%$ de los pacientes se observó dicho signo clínico. Para el tiempo de cicatrización de la EAR con el uso del croton lechleri, en los sujetos de estudio se observó que, al tercer día, la lesión presentó características clínicas de cicatrización, mientras que, en el segundo día, el $50 \%$ de los pacientes presentó características del proceso de cicatrización.
Con referente a los niveles de leucocitos y PCR, antes y después de la aplicación de los medicamentos, con el uso del eugenol se encontró diferencia significativa en la cuantificación de leucocitos y PCR con un valor de $p=0,04$; de igual manera existe diferencia significativa antes y después de la aplicación del croton lechleri cuyo valor de $p=0,02$ para cuantificación de leucocitos y $p=0,03$ para PCR. En el G.C. no se evidenció un valor de $p>0,05$ (ver tabla 5).

Al aplicar la prueba no paramétrica Kruskal Wallis, esta mostró un valor de $p=0,00$, donde se afirma que existen diferencias significativas en los tres grupos de estudio en el efecto antiinflamatorio para el tratamiento de la EAR (ver tabla 6).

Al aplicar la prueba no paramétrica, $U$ de Mann Whitney, esta mostró un valor de $p=0,602$; lo que expresa que no existen diferencias significativas sobre el efecto antiinflamatorio del croton lechleri y el eugenol, en el tratamiento de la EAR de ambos grupos de estudio. Ambos agentes son efectivos para dichas lesiones, por lo tanto se acepta la hipótesis nula donde se asevera que no existe diferencia en la efectividad del croton lechleri y el eugenol como antiinflamatorio en el tratamiento de la EAR.

\section{DISCUSIÓN}

La EAR es una condición complicada y su etiología precisa sigue siendo desconocida. La alta frecuencia, la distribución mundial, el amplio rango de edad, el carácter recurrente de la enfermedad y la disminución de la calidad de vida de los pacientes con EAR han conducido a una gran cantidad de investigaciones

Tabla 5. $T$ de Student: Niveles de Leucocitos y PCR antes y después de la aplicación de los medicamentos

\begin{tabular}{|c|c|c|c|c|c|c|c|c|c|}
\hline & \multicolumn{2}{|c|}{$\begin{array}{c}\text { Eugenol } \\
\text { Media }\end{array}$} & \multirow[b]{2}{*}{$p$} & \multicolumn{3}{|c|}{$\begin{array}{c}\text { Croton Lechleri } \\
\text { Media }\end{array}$} & \multicolumn{3}{|c|}{$\begin{array}{c}\text { Agua destilada } \\
\text { Media }\end{array}$} \\
\hline & Antes & Despues & & Antes & Despues & $p$ & Antes & Despues & $p$ \\
\hline Leucocitos & 11,850 & 8,920 & 0,04 & 11,640 & 8,043 & 0,02 & 11,450 & 10,241 & 0,8 \\
\hline PCR & 8,00 & 4,00 & 0,04 & 7,00 & 2,00 & 0,03 & 7,00 & 4,00 & 0,69 \\
\hline
\end{tabular}


Tabla 6. Efectividad antiinflamatoria del Eugenol frente al Croton Lechleri

\begin{tabular}{lc}
\hline \multicolumn{1}{c}{ Estadísticos de Prueba } & $\boldsymbol{p}$ \\
\hline Kruskal Wallis: Entre los tres grupos & 0,000 \\
U de Mann Whitney: Entre Croton Lechleri y Eugenol & 0,602 \\
\hline
\end{tabular}

sobre la etiología y el tratamiento de esta afección. Se han evaluado diversas modalidades de tratamiento, agentes tópicos y sistémicos en diferentes países durante muchos años ${ }^{(13)}$.

Como es sabido, el tratamiento de primera línea de EAR siempre debe comenzar con medicamentos tópicos (14); como enjuagues orales, geles, parches, tabletas o películas adhesivas que brinden efectos antisépticos, antiinflamatorios y analgésicos ${ }^{(15,16) \text {. }}$

Clínicamente, la EAR se presenta en tres variedades: forma menor, mayor o herpetiforme, las que se diferencian por las características clínicas de las lesiones y su tamaño. La más común es el afta menor, que no supera los $6 \mathrm{~mm}$ de diámetro y pueden aparecer, una o varias, en diferentes regiones de la boca, simultáneamente. Las aftas mayores, con más de $6 \mathrm{~mm}$, generalmente no aparecen más de dos al mismo tiempo. Por último, la forma herpética, similar a un herpes, que erupciona a partir de muchos puntos repartidos por el cuerpo ${ }^{(17)}$.

En el estudio se encontró mayor número de casos de la forma menor de la EAR con un 93,3\% del total; dicho resultado coincide con lo hallado por Raissouni quien encontró un $84 \%$ de pacientes con formas menores. El eugenol redujo todas las características clínicas de la inflamación de la lesión en cuatro días; mientras que el dolor desapareció en el $100 \%$ de los pacientes al segundo día después del tratamiento. Ayala concluyó que el eugenol tiene realmente un potente poder analgésico en las aftas; lo cual se pudo percibir, sobre todo en los 2 minutos posteriores de la aplicación, con un 95,2\% de los casos y a los 4 días con un $100 \%$ de los mismos.

Además del dolor, el tiempo de cicatrización y el tiempo de desaparición del eritema, el eugenol mostró ser efectivo para el tratamiento de la EAR cesando todas las características clínicas al cuarto día de aplicado; resultados que concuerdan con lo evidenciado por la observación clínica de Ayala donde se observó que el efecto antiinflamatorio fue favorable, al reducirse el diámetro de la lesión en el periodo comprendido entre el tercer y quinto día. Otros agentes antiinflamatorios utilizados por Bhalang et al., informaron una mayor efectividad de $0,1 \%$ de acetonido de triamcinolona (TA) que el acemannan, un polisacárido extraído de aloe vera, en el tratamiento de la ulceración aftosa oral ${ }^{(18)}$. Comparando TA 0,025\% con clorhexidina aplicada tópicamente $0,12 \%$, cubierta por una barrera de cianoacrilato de isobutilo, en el tratamiento de EAR, un estudio clínico controlado y aleatorizado encontró una diferencia muy significativa en la reducción de la intensidad y la percepción del dolor en diferentes días, cuando los dos grupos con medicación se compararon con el grupo control; pero la diferencia no fue significativa cuando se compararon los dos medicamentos ${ }^{(19,20)}$.

Los resultados del presente estudio demuestran que la aplicación tópica de croton lechleri y el eugenol son efectivos como agentes anitiinflamatorios en el tratamiento de la EAR. El croton lechleri o sangre de grado mostró mejor comportamiento clínico que el eugenol. Ambos disminuyen la intensidad del dolor y aceleran la curación de la úlcera sin efectos secundarios y con un procedimiento accesible y fácilmente aplicable. Por lo tanto, ambos podrían ser un agente terapéutico tópico seguro y bien tolerado en la práctica clínica del tratamiento de la EAR.

Se concluye indicando que los resultados del presente estudio demuestran que la aplicación tópica de croton lechleri y el eugenol son efectivos en el tratamiento de la EAR. En las características clínicas de la EAR según tipo predominaron las aftas menores, en su forma de lesión redonda; la cual se presentó con mayor frecuencia; además, y de acuerdo a la localización, se presentó en varias áreas de la mucosa oral. El tiempo de desaparición de las características clínicas de la EAR con el uso de eugenol fue en promedio tres días, siendo para el dolor dos días, eritema tres días y tiempo de cicatrización cuatro días.

El tiempo de desaparición de las características clínicas de la EAR con el uso del croton lechleri fue en promedio tres días, siendo para el dolor, eritema y tiempo de cicatrización tres días. En cuanto a los niveles de leucocitos y PCR antes y después de la aplicación del eugenol se encontró diferencia significativa con un valor de $p=0,04$ al aplicar la prueba t de Student para muestras relacionadas. En cuanto a los niveles de leucocitos y PCR antes y después de la aplicación del croton lechleri se encontró diferencia significativa con un valor de $p=0,02$ al aplicar la prueba $t$ de Student para muestras relacionadas; finalmente no se evidenciaron efectos secundarios al aplicar el eugenol y croton lechleri como agente terapéutico tópico en la EAR. 


\section{REFERENCIAS BIBLIOGRÁFICAS}

1. Lynch B. Medicina Bucal de Burket. 9a ed. México, D. F.: McGraw-Hill Interamericana; 1996.

2. American National Standard/American Dental Association Specification No. 30 for dental zinc oxide-eugenol cements and zinc oxide non-eugenol cements. ANSI/ ADA Specification; 1990.

3. Chami N, Chami F, Bennis S, Trouillas J, Remmal A. Antifungal Treatment with Carvacrol and Eugenol of Oral Candidiasis in Immunosuppressed Rats. Braz J Infect Dis. 2004; Jun; 8 (3): 217-26.

4. Risco E, Iglesias J, Cañigueral S. Interés Terapéutico del Látex de Croton lechlerisp. En: 12éme Forum De Natura Rerum París; 2001.

5. Briseño B, Willers Lausen B. Root canal sealer cytotoxity on human gingival fibroblasts. JJ Endodon 1990; 16: 383.

6. Giunta, J. Patología Bucal. 3a ed. México, D. F.: McGrawHill Interamericana; 1997.

7. Miller $M$, Macnaughton $W$, Zhang $X$, Thompson J, Charbonnet R, Bobrowski P, et al. Treatment of gastric ulcers and diarrhea with the Amazonian herbal medicine sangre de grado. Am J Physiol Gastrointest Liver Physiol. 2000 Jul; 279 (1): 192-200.

8. Neill D, Palacios W. Árboles de la Amazonia Ecuatoriana, lista preliminar de especies. Quito: Ministerio de Agricultura y Ganadería, Dirección Nacional Forestal; 1989.

9. Persinos G, Blomster R, Blake D, Farnsworth N. South American plants II: taspine isolation and Anti-inflammatory activity. J Pharm Sci. 1979 Jan; 68(1): 124-6.

10. Medrano C. Reacción del tejido subcutáneo a los cementos de obturación a base de Bálsamo de Perú y Sangre de Grado en ratones Suizos [Tesis para optar el grado de bachiller]. Lima: Facultad de Estomatología. Universidad Particular Cayetano Heredia; 1985.

11. Zaravia R. Reacción antiinflamatoria del tejido conjuntivo al cemento de obturación de conductos a base $\mathrm{C}$.
Lechleri ("Sangre de Grado") en ratas de cepa Holtzman. [Tesis para optar el grado de bachiller]. Lima: Facultad de Estomatología. Universidad Peruana de Ciencias Informáticas; 1985.

12. Gilbert B, Wyde P, Wilson S, MeyersonL. SP-303 samllparticle aerosol treatment of influenza A. virus infection in mice and respiratory syncytial virus infection in cotton rats. Antiviral Res. 1993 May; 21(1): 37-45.

13. Akintoye SO, Greenberg MS. Recurrent Aphthous Stomatitis. Dent Clin North Am. 2014; 58(2):281-297.

14. Scully C, Porter S. Enfermedad de la mucosa oral: estomatitis aftosa recurrente. Br J Oral Maxillofac Surg. 2008; 46: 198-206.

15. Baccaglini L, Lalla RV, Bruce AJ, JC Sartori-Valinotti, MC Latortue, Carrozzo M. Leyendas urbanas: estomatitis aftosa recurrente. Dis. Oral. 2011; 17(8): 755-770.

16. Meng W, Dong Y, Liu J, Wang Z, Zhong X, Chen R. Evaluación clínica de las películas con adhesivo oral de amlexanox en el tratamiento de la estomatitis aftosa recurrente y comparación con tabletas orales de amlexanox: un estudio aleatorizado, controlado con placebo, ciego. ensayo clínico multicéntrico. Ensayos. 2009; 10: 30.

17. Castillo A, Pérez A, Guntiñas MV. Uso del inmunoferón en el tratamiento de la estomatitis aftosa recurrente en niños. Rev Cubana Estomatol. 2006; 43(3).

18. Bhalang K, Thunyakitpisal P, Rungsirisatean N. Acemannan, a Polysaccharide Extracted from Aloe vera, Is Effective in the Treatment of Oral Aphthous Ulceration. J Alternar Complemento Med. 2013; 19(5): 429-34.

19. Quijano D, Rodríguez M. Corticosteroides tópicos en la estomatitis aftosa recurrente. Acta Otorrinolaringol Esp. 2008; 59(6): 298-307.

20. Miles DA, Bricker SL, Razmus TF, Potter RH. Triamcinolone acetonide versus clorhexidine for treatment of recurrent stomatitis. Oral Surg Oral Med Oral Pathol. 1993 Mar; 75(3): 397-402. 